\title{
Spectral ellipsometry of binary optic gratings
}

\author{
Jaromir Pistora ${ }^{1,2}$, Tomuo Yamaguchi', Jaroslav Vlcék ${ }^{2}$, Jan Mistrik', Masahiro Horie ${ }^{3}$, \\ Vasilis Smatko ${ }^{4}$, Eva Kovácova ${ }^{4}$, Kamil Postava $^{2}$, Mitsuru Aoyama ${ }^{1}$ \\ ${ }^{1}$ Research Institute of Electronics, Shizuoka University, 3-5-1 Johoku, Hamamatsu 432, Japan, \\ e-mails: rjpisto@ipc.shizuoka.ac.jp, rstyama@rie.shizuoka.ac.jp. \\ ${ }^{2}$ Department of Physics, Department of Mathematics, Technical University Ostrava, 17. listopadu 15, \\ 70833 Ostrava-Poruba, Czech Republic, e-mails: kamil.postava@vsb.cz, jaroslav.vlcek@vsb.cz. \\ ${ }^{3}$ Dainippon Screen Manufacturing Company Ltd., Kyoto, Japan. \\ ${ }^{4}$ Institute of Electrical Engineering, Slovak Academy of Sciences, Dúbravská cesta 9, \\ Bratislava, Slovak Republic.
}

\begin{abstract}
The coupled wave method (CWM) has been applied to the description of electromagnetic wave propagation in binary optic gratings. The electromagnetic field and the permittivity profile are expanded into two-fold Fourier series. The reflection coefficients of 2D periodical structures have been specified and the ellipsometric angles of the shapes discussed have been computed. The theoretical results are compared with experimental data obtained on $\mathrm{SiO}_{2}$ square dots on Si substrate. The measurements were performed using computer controlled four zone null ellipsometer in spectral range from $240 \mathrm{~nm}$ to $700 \mathrm{~nm}$. The influence of $\mathrm{SiO}_{2}$ ultrathin oxidation layer and thickness of dots on spectral ellipsometric angles is also discussed.
\end{abstract}

Keywords: lamellar grating, spectral ellipsometry.

\section{Introduction}

The lamellar and binary optic periodical structures bring the possible new applications in the integrated optics, magnetooptic memories, and in sensor technique [1], [2]. The special attenuation is at the present devoted to metallic gratings [3]-[5], magnetic dots and nanostructures [6], [7].

There are several different approaches to solve the problem of electromagnetic field propagation in periodical systems. An efficient method to calculate the lattice sums for 1D periodic array of line sources [8], differential theory for anisotropic gratings [9], [10], and recursive matrix algorithms for modeling layered diffraction gratings [11] have been presented. The reformulated CWM has been described and 
applied to isotropic and anisotropic periodical structures (including metallic gratings) [12]-[14] and the systematic comparison of the scalar diffraction analysis and CWM has been published for two dimensional periodic systems [15]. A rigorous differential method has been used to study the influence of the height of grating on the diffraction hysteresis loop diagram [16].

The phase modulated spectroscopic ellipsometry (PMSE) is an excellent experimental technique for specification of optical and geometrical parameters of thin films and multilayer dielectric coatings. This technique has been applied ex situ and in situ for the growth control of transparent films with varying composition [17]-[19]. Recent advances in PMSE instrumentation are summarized in [20].

In the present paper, we specify the possibilities of implementing the CWM as the Fourier modal method (FMM) for the description of electromagnetic wave propagation in $2 \mathrm{D}$ gratings. The CWM model ellipsometric parameters of diffracted waves were compared with experimental results. The measurements have been performed by the PMSE on periodical structures completed by square $\mathrm{SiO}_{2}$ dots located on $\mathrm{Si}$ substrate.

\section{Theoretical}

The coordinate system is introduced as in Fig. 1. Generally, we assume that the grating structure is created by binary modulated (in $x-y$ plane) layers indexed $v=1, \ldots, K$. The thickness of the layer numbered $v$ is $h^{(v)}$. The multilayer periodic system discussed is sandwiched between two semi-infinitive isotropic regions. One of them is the substrate $\left(v=K+1, z \geq \sum_{v=1}^{K} h^{(v)}\right)$; the second region (superstrate) is specified by $v=0, z \leq 0$.

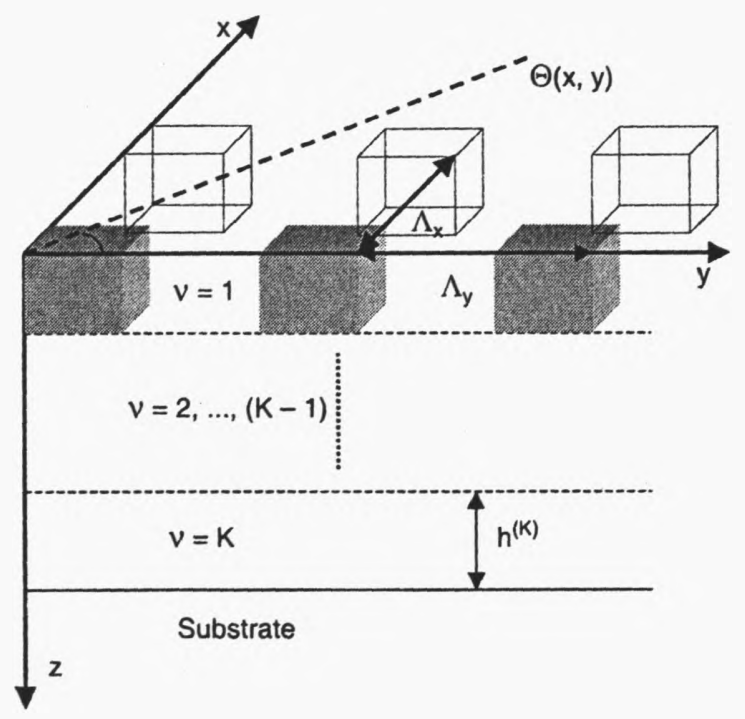

Fig. 1. Periodic structure, coordinate system, and plane of incidence geometry. 


\subsection{Field in the superstratum region}

\subsubsection{Incident field}

Incident monochromatic plane wave with free space wavelength $\lambda$ propagates in the homogeneous isotropic superstrate with refractive index $n^{(0)}$. The wave vector forms an angle $\varphi$ relative to $z$-axis and these two directions define the incidence plane. Its deviation from the $y$-axis is denoted by $\theta$. Thus, the wave vector is expressed in the form

$$
\mathbf{k}_{0}^{(0)+}=k_{0} n^{(0)}(\sin \theta \sin \varphi, \cos \theta \sin \varphi, \cos \varphi)=k_{0}\left(k_{1}, k_{2}, n^{(0)} \cos \varphi\right)
$$

where $k_{0}=2 \pi / \lambda$.

The polarization state of incident field is generally elliptical and defined by the angle $\vartheta$ between unit-amplitude electrical field vector

$$
\mathbf{u}_{i}=(\sin \theta \cos \varphi \cos \vartheta+\cos \theta \sin \vartheta, \cos \theta \cos \varphi \cos \vartheta-\sin \theta \sin \vartheta,-\sin \theta \cos \vartheta)
$$

and the incidence plane. Thus, we put $\vartheta=0$ for $p$-polarization or $\vartheta=\pi / 2$ for $s$-polarization. The electric field with the complex amplitude $E_{i}^{(0)}$ of the incident wave is then given by

$$
\mathbf{E}_{i}(\mathbf{r})=E_{i}^{(0)} \mathbf{u}_{i} \exp \left[i \omega t-i\left(\mathbf{k}_{0}^{(0)+} \cdot \mathbf{r}\right)\right]
$$

where $\mathbf{r}=\mathbf{r}(x, y, z)$. It is more convenient to normalize the space coordinates by the wavenumber in the free space $\mathbf{k}_{0}$. Let $\mathbf{x}$ denote the modified coordinates as $\mathbf{x}=\left(x_{1}, x_{2}, x_{3}\right)=k_{0}(x, y, z)$. In this case, we can write

$$
\mathbf{E}_{i}(\mathbf{x})=E_{i}^{(0)} \mathbf{u}_{i} \exp \left[i \omega t-i n^{(0)}\left(x_{1} \sin \theta \sin \varphi+x_{2} \cos \theta \sin \varphi+x_{3} \cos \varphi\right)\right] .
$$

\subsubsection{Diffracted field}

The diffracted fields outside the modulated region may be expressed in the form of Rayleigh expansions. For a doubly periodic element the reflected electric field may therefore be written as (the term $z_{0}=\sqrt{\mu_{0} / \varepsilon_{0}}$ - free space impedance - is incorporated in the field $\mathbf{H}$; the time dependence $\exp \{i \omega t\}$ is assumed but formally suppressed in the next one)

$$
\begin{aligned}
& \mathbf{E}^{(0)}\left(x_{1}, x_{2}, x_{3}\right)=\sum_{q} u_{q}^{(0)} \mathbf{E}_{q}^{(0)}\left(x_{1}, x_{2}, x_{3}\right) \exp \left(-i \mathbf{k}_{q}^{(0)} \cdot \mathbf{x}\right), \\
& \mathbf{H}^{(0)}\left(x_{1}, x_{2}, x_{3}\right)=\sum_{q} u_{q}^{(0)} \mathbf{H}_{q}^{(0)}\left(x_{1}, x_{2}, x_{3}\right) \exp \left(-i \mathbf{k}_{q}^{(0)} \cdot \mathbf{x}\right)
\end{aligned}
$$

with amplitude coefficients $u_{q}^{(0)}$ and wave vectors 


$$
\mathbf{k}_{q}^{(0)}=\mathbf{k}_{m n q}^{(0)}=\left(k_{1}+m \lambda / \Lambda_{x}, k_{2}+n \lambda / \Lambda_{y}, \gamma_{q}^{(0)}\right)=\left(k_{1 m}, k_{2 n}, \gamma_{q}^{(0)}\right)
$$

where the parameter $q=1, \ldots, 4 d$, while $4 d=4(2 M+1)(2 N+1)$. Here $M$ and $N$ specify the finite numbers of harmonic components in truncated Fourier series [21].

\subsection{Fourier modal method}

In the FMM, both the electromagnetic fields and the permittivity function are expanded into two-fold Fourier series, thereby the boundary value problem for a system of partial differential equations is reformulated to an algebraic eigenvalue problem of the 4-dimension. According to the Floquet-Bloch theorem in double periodic medium with period dimensions $\Lambda_{x}, \Lambda_{y}$ the transformed expressions for amplitude components take the form

$$
\begin{aligned}
& E_{j}^{(v)}\left(x_{1}, x_{2}, x_{3}\right)=\sum_{m} \sum_{n} \sum_{q} u_{q}^{(v)} e_{j m n q}^{(v)} \exp \left[--i\left(k_{1 m} x_{1}+k_{2 n} x_{2}+\gamma_{q}^{(v)} x_{3}\right)\right] \\
& H_{j}^{(v)}\left(x_{1}, x_{2}, x_{3}\right)=\sum_{m} \sum_{n} \sum_{q} u_{q}^{(v)} h_{j m n q}^{(v)} \exp \left[-i\left(k_{1 m} x_{1}+k_{2 n} x_{2}+\gamma_{q}^{(v)} x_{3}\right)\right] .
\end{aligned}
$$

The dependence $x_{3}$ characterized by $\gamma_{q}^{(v)}$, which does not follow from the Floquet theorem, has been included.

The Fourier transform of the permittivity tensor components can be written as

$$
\varepsilon_{i j}=\sum_{k} \sum_{l} c_{i j, k l} \exp \left[-i\left(\lambda k x_{1} / \Lambda_{x}+\lambda l x_{2} / \Lambda_{y}\right)\right]
$$

where $k, l$ are from the set of integers. Substituting the above expansions into Maxwell's equations we obtain an infinite-dimensional algebraic system for unknown Fourier coefficients $e_{j m n}, h_{j m n}(j=1,2,3)$, where we apply the multiplication rule for the permittivity and electrical field Fourier series. We introduce in each layer the column vectors of Fourier coefficients $\mathbf{e}_{j}$ and $\mathbf{h}_{j}$ of the dimension $d=(2 M+1)(2 N+1)$. On the basis of tangential field component continuity we can create the single vector $\mathbf{g}=\left(\mathbf{e}_{1}, \mathbf{h}_{2}, \mathbf{e}_{2}, \mathbf{h}_{1}\right)$. Eliminating coefficients $\mathbf{e}_{3}$ and $\mathbf{h}_{3}$ from governing algebraic system we obtain eigenvalue problem. Its solution leads to the eigenvalues $\gamma_{q}$ (propagation constants) and square matrix $\mathbf{D}=\left(\mathbf{g}_{q}\right)$ of corresponding column eigenvectors (eigenpolarizations) in the relevant layer. Denoting by $\mathbf{u}^{(0)}, \mathbf{u}^{(K+1)}$ the 4-dimensional vectors of amplitude coefficients in the superstrate and substrate, we can express the coupling condition of all layers as

$$
\mathbf{u}^{(0)}=\mathbf{M} \cdot \mathbf{u}^{(K+1)}, \quad \mathbf{M}=\left(\mathbf{D}^{(0)}\right)^{-1} \cdot \prod_{v=1}^{K} \mathbf{S}^{(v)} \cdot \mathbf{D}^{(K+1)}
$$


where any layer of the thickness $h^{(v)}$ contributes to the matrix $\mathbf{M}$ by the term $\mathbf{S}^{(v)}=$ $\mathbf{D}^{(v)} \cdot \mathbf{P}^{(v)} \cdot\left(\mathbf{D}^{(v)}\right)^{-1}$. The matrix $\mathbf{D}^{(v)}$ is composed from 4-dimensional column vectors of the form $\left(\mathbf{e}_{1} \mathbf{h}_{2} \mathbf{e}_{2} \mathbf{h}_{1}\right)^{\mathrm{T}}$.

Reflection properties of the measured samples are described by complex ellipsometric ratio $\rho^{(0)}$ (we assume that the incident light is linearly polarized at azimuth $\theta=\pi / 4)$. If $u_{p p}$ and $u_{s s}$ are amplitude coefficients of $p$ - and $s$-polarized wave, then

$$
\rho_{Q}^{(0)}=\frac{u_{p p, Q}^{(0)-}}{u_{s s, Q}^{(0)-}}
$$

where $Q=1, \ldots, 4 d$; the sign "-“" respects the negative direction considering $z$-axis. In the case of magnetooptical gratings

$$
\rho_{Q}^{\prime} \stackrel{(0)}{Q}=\frac{u_{p p, Q}^{(0)-}+u_{s p, Q}^{(0)-}}{u_{s s, Q}^{(0)-}+u_{p s, Q}^{(0)-}} .
$$

The application of the last two relations makes it possible to specify the ellipsometric angles $\psi$ and $\Delta$ for isotropic and anisotropic binary gratings, too. For a structure with magnetic ordering this process has been demonstrated in [21]. Knowing the dispersion curves of permittivity we can analyze the spectral ellipsometry data.

The algorithm derived was implemented in the Matlab6.0 code. The square sample configuration with the periodicity $\Lambda_{x}=\Lambda_{y}$ leads to equal truncation orders $M=N$ which implies global dimension of eigenvalue problem of $4(2 M+1)^{2}$. For instance, it gives 1444 modes for $M=9$ (maximal value of applied truncation order). An error less than $10^{-7}$ was obtained in the numerical stability test in the case of lossless media for both polarization states [21], [22].

The free parameters that may be utilized in the synthesis are: i) the grating period $\Lambda_{x} \times \Lambda_{y}$, ii) the relief depth $h$, iii) the buffer thickness, iv) the indices of refraction of substrate, thin films, and periodic structure, and v) the parameters that define the transverse structure of the features.

\section{Experimental}

The experiments were performed using computer controlled four zone null ellipsometer with polarizer-sample-compenzator-analyzer (PSCA) configuration in the spectral region from 240 to 700 nanometers (see Fig. 2). A Xe-Hg lamp was used as a light source. A $10 \mathrm{~cm}$ double-grating monochromator combinated with a photomultiplier was employed as a null detector. Rochon quartz prism polarizer and analyzer were rotated by stepping motors to adjust a minimal intensity. A four total reflection -type Fresnel rhomb made up of two fused silica rods was used as an achromatic quarter-wave retarder. The four-zone averaging for two compensator azimuth angles $\pm 45^{\circ}$ gives high precision measurements insensitive to azimuth angle errors and imperfections of the compensator, polarizer and analyzer. Absence of systematic errors 


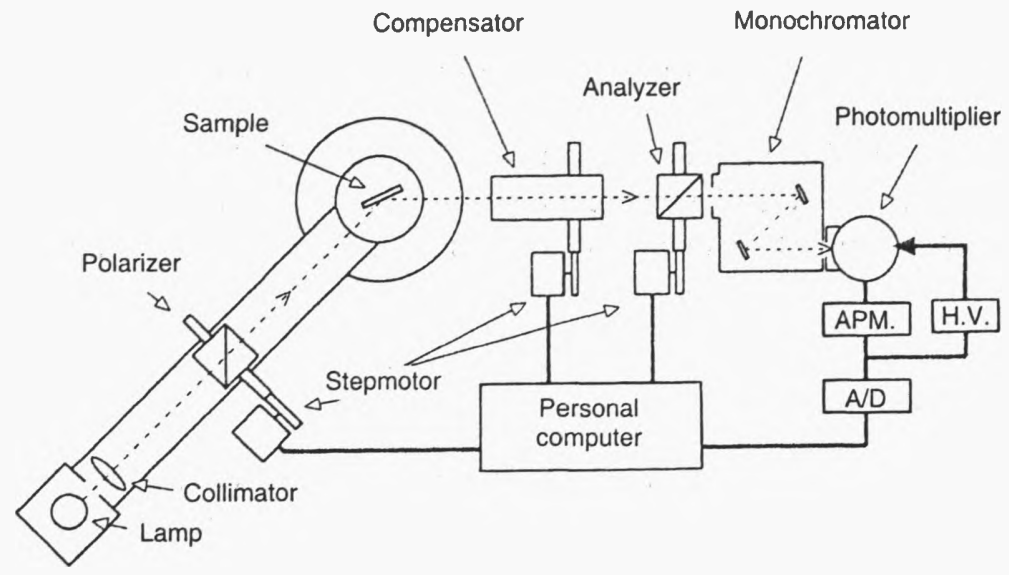

Fig. 2. Experimental arrangement for spectral ellipsometry.

is essential for a reliable determination of unknown parameters. The experiments have been performed under the incidence angles ranging from 65 to $72^{\circ}$. The plane of incidence was adjusted to be parallel or diagonal to the dot configuration.

The samples $\left(\mathrm{SiO}_{2}\right.$ square dots $50 \times 50 \mathrm{~mm}$ with the same periodicity of $120 \mu \mathrm{m}$ in $x$-and $y$-direction) were prepared using standard chemical vapour deposition on single -crystal silicon wafers.

\section{Results and discussion}

The experimental results and modeling data are demonstrated in the collection of figures. The influences of ultrathin oxidation layer, the geometrical position of incidence optical plane, the angle of light beam incidence, the dot thickness, and the

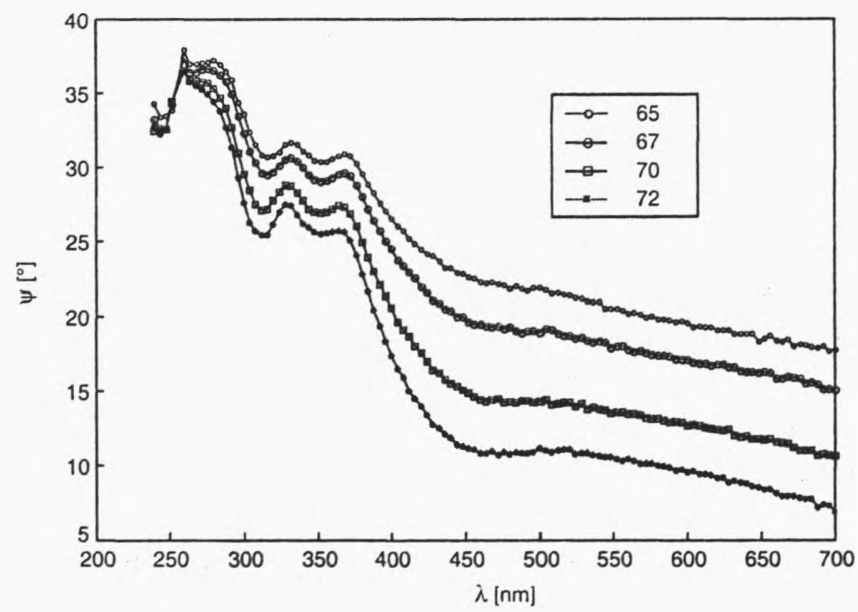

Fig. 3. Spectral dependences of ellipsometric parameters $\psi$ for binary grating at different incidence angles. 


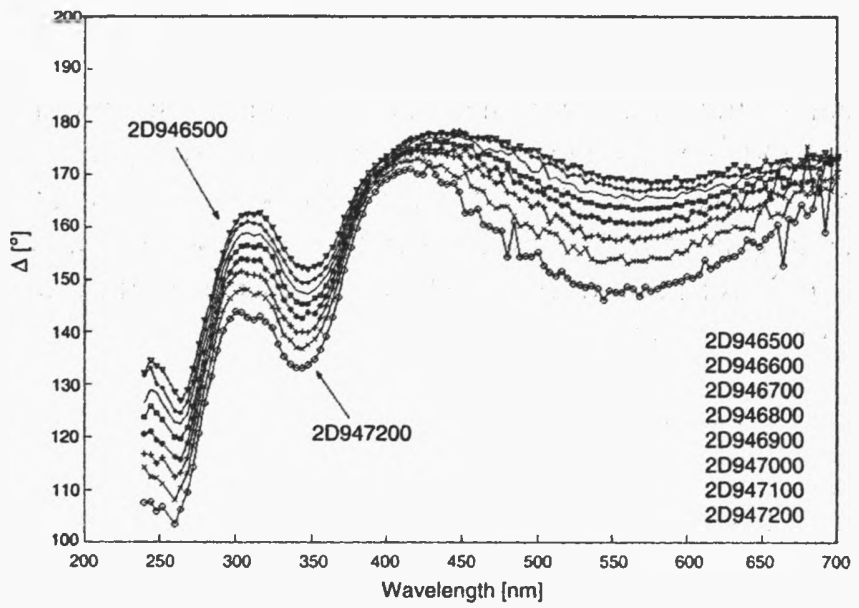

Fig. 4. Spectral dependences of ellipsometric parameters $\Delta$ for binary grating at different incidence angles.

value of refractive index have been analyzed in detail. Finally, a comparison has been made of the experimental values of spectral ellipsometry with the model ellipsometric angles computed by CWM approach.

The spectral dependences of ellipsometric angles for binary gratings for the incidence angles $\varphi_{i}$ from 65 to $72^{\circ}$ are collected in Figs. 3 and 4 . All measurements in this case have been performed at the geometrical configuration when the plane of incident light was parallel to dot edges. The angles $\varphi_{i}$ have been chosen to be close to the values obtained when the module $\left|r_{p}\right|$ converges to minimal value. In this area, the sensitivity of experimental setup attains maximum. It is worth noting that the ellipsometric angles decrease with increasing value of angles $\varphi_{i}$. This tendency

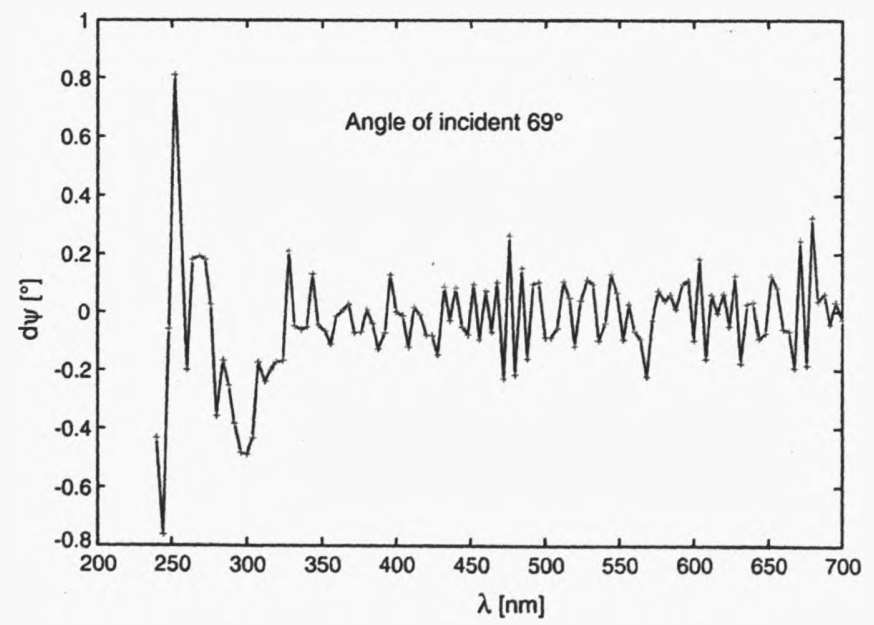

Fig. 5. Ellipsometric differences of $\psi$ generated by incident plane rotation (parallel or diagonal geometry to grating dot edges). 
indicates that the measurements were made at the incidence angles less than "pseudo" -Brewster angle.

The theoretical model predicts the practically neglected effect of the mutual position of the incidence plane and dot geometry (in the first case, the plane of incidence was supposed to be parallel with dot edges; in the second case this configuration was oriented diagonally at $45^{\circ}$ ). The differences between relevant values have been found to be less than $0.2^{\circ}$ for parameters $\psi$. The experimental outputs are collected for the incident angle of $69^{\circ}$ in Fig. 5 .

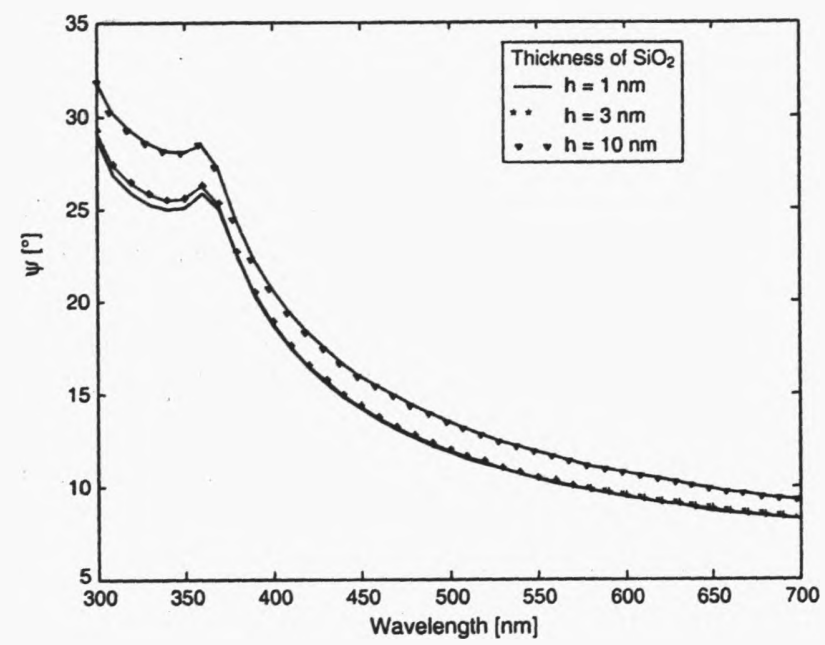

Fig. 6. Influence of ultrathin $\mathrm{SiO}_{2}$ layer deposited on $\mathrm{Si}$ substrate on spectral ellipsometric angles $\psi$ for planar structure.

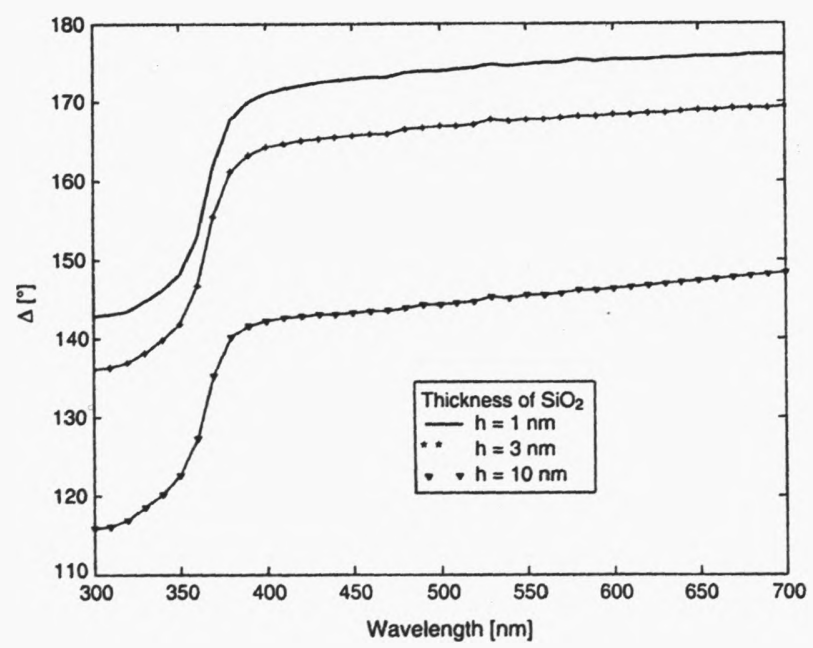

Fig. 7. Influence of ultrathin $\mathrm{SiO}_{2}$ layer deposited on $\mathrm{Si}$ substrate on spectral ellipsometric angles $\Delta$ for planar structure. 
Because the dots are located on silicon wafers the effect of ultrathin oxidation layer on the top of substrate has to be analyzed.

The shifts of ellipsometric angles generated by this oxidation process have been computed for planar structures. The results for three different thickness values of continuous layers are demonstrated in Figs. 6 and 7. The oxidation process causes an increase of parameters $\psi$; for values of angle $\Delta$ we can observe the opposite tendency. These changes are in very good agreement with published data [23].

Experimental angles $\Delta$ for the planar system and grating structure are compared in Fig. 8. The values of ultrathin oxidation layers of $\mathrm{SiO}_{2}$ have been determined by fitting spectral ellipsometric data for the case of thin film - substrate model.

A comparison of the values measured by spectral ellipsometry and those obtained from theoretical model for binary gratings is shown in Figs. 9, 10. The experimental dependence of $\psi$ (angle of incidence of $71^{\circ}$ ) shows within the measured wavelength interval the first maximum at about $260 \mathrm{~nm}$; and the first minimum located close to $315 \mathrm{~nm}$. From the wavelength of $370 \mathrm{~nm}$ onwards we can observe a continuous decrease up to the limit value of $9^{\circ}$ at $700 \mathrm{~nm}$. The same tendency is exhibited by the computed shape for $200 \mathrm{~nm}$ thick $\mathrm{SiO}_{2}$ dots. The parameters $\Delta$ are characterized by the first minimum at $256 \mathrm{~nm}$ followed by maximum (at $306 \mathrm{~nm}$ ); the position of the second minimum lies around $340 \mathrm{~nm}$. The next maximum can be registered about $425 \mathrm{~nm}$. The dependence computed for $200 \mathrm{~nm} \mathrm{SiO}{ }_{2}$ dots differs from the measured data significantly in the band from 500 to $700 \mathrm{~nm}$.

The permittivity dispersion curve of dots material for computing has been represented by dielectric function of $\mathrm{SiO}_{2}$ (Sellmeier's equation)

$$
\varepsilon(\lambda)=1+\frac{A \lambda^{2}}{\lambda^{2}-B^{2}}
$$

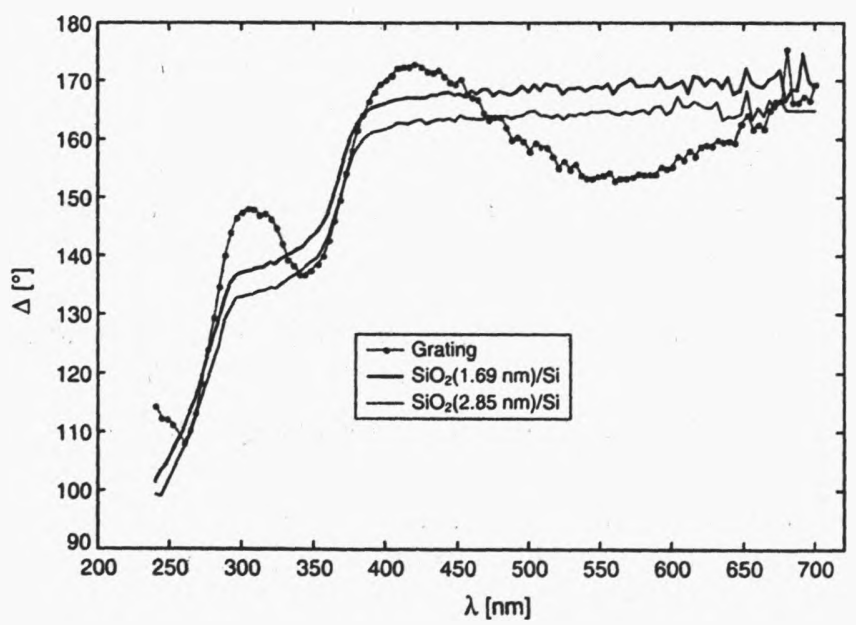

Fig. 8. Experimental values of spectral ellipsometric angles $\Delta$ for planar structure with $\mathrm{SiO}_{2}$ layer fitting and $\Delta$ shape modulation by periodic structure. 


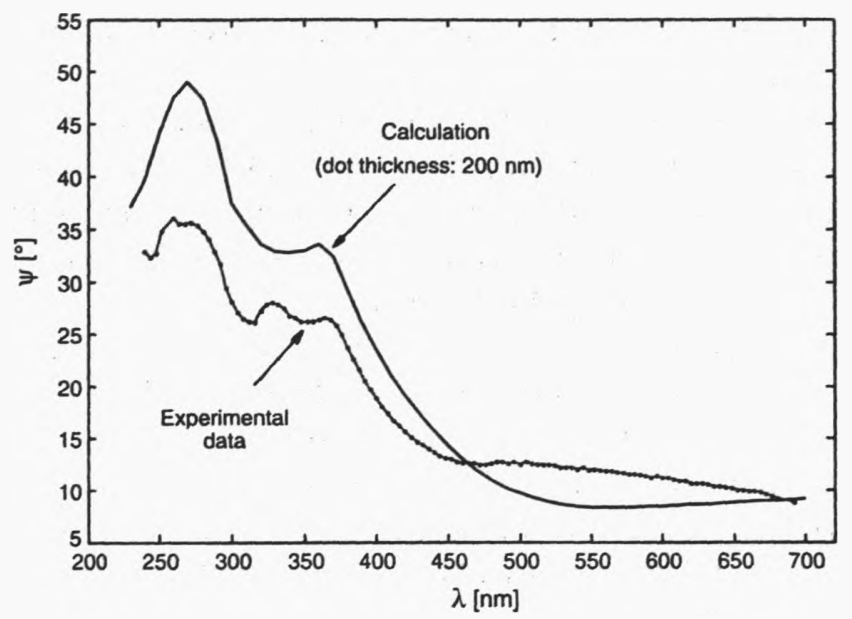

Fig. 9. Comparison of measured spectral angles $\psi$ with computed relevant ellipsometric values ( $200 \mathrm{~nm}$ dot thickness in modeling).

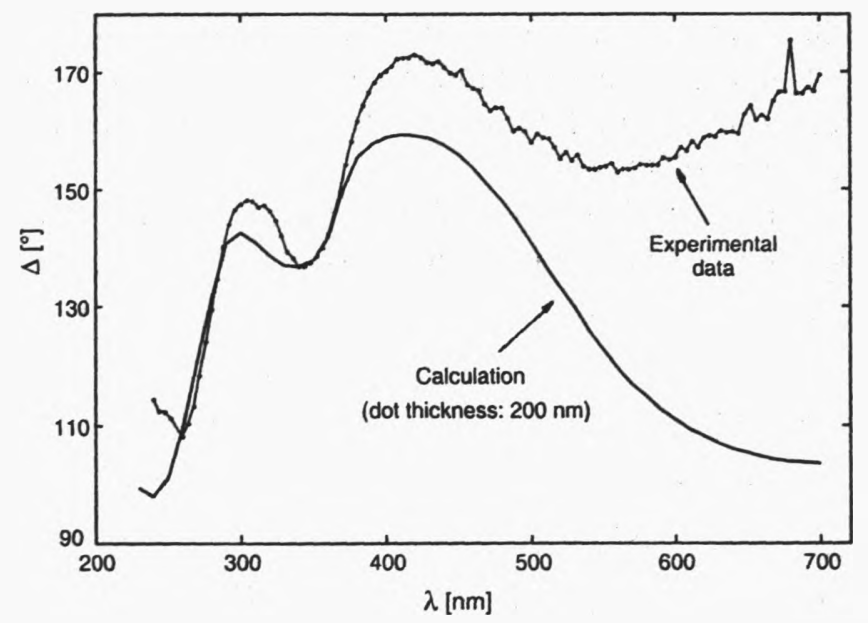

Fig.10. Comparison of measured spectral angles $\Delta$ with computed relevant ellipsometric values (200 $\mathrm{nm}$ dot thickness in modeling).

where $A=1.1336$ and $B=92.61 \mathrm{~nm}$. The optical parameters of $\mathrm{Si}$ have been taken from PaliK [24].

The differences between experimental and theoretical data in Figs. 9, 10 can be explained as follows:

- diffracted order superposition (the result of $\Lambda / \lambda$ relationship);

$-\mathrm{SiO}_{2}$ buffer thickness is less than $4 \mathrm{~nm}$ which was included in the model;

- thickness of $\mathrm{SiO}_{2}$ dots is greater than $200 \mathrm{~nm}$;

- limitation of the total number of Rayleigh orders during computation; 
- thickness uniformity of $\mathrm{SiO}_{2}$ buffer film (areas between dots and below them);

- number of dots covered by incident light beam (integral factor).

The previously presented papers discuss the ability of CWM method for the specification of electromagnetic field distribution in periodical structures up to periodicity $\Lambda_{x}\left(\Lambda_{y}\right)=10 \lambda$ [13]. If we retain Rayleigh orders $L_{x}$ in the $x$-direction and orders $L_{y}$ in the $y$-direction, a total number $L=L_{x} L_{y}$ of orders has to be included in the analysis. The authors in [13] say that the convergence is somewhat better for the longer period, but in the inclusion of $L=7-9$ orders provides an accuracy of two decimal places, whereas three correct decimal places are obtained if $L=13-17$. The geometry of our samples is in stark contrast to the above-mentioned relation between periodicity and wavelength because $\Lambda_{x} / \lambda \in\langle 200,500\rangle$.

The angle difference of diffracted light in the frame of the 1st order for limit experimental wavelengths $\left(240 \mathrm{~nm}\right.$ and $700 \mathrm{~nm}$, respectively) is only $0.66^{\circ}$ for incidence angle of $70^{\circ}$. The diffracted beam angle difference for zero and 1 st order for limit experimental wavelengths $240 \mathrm{~nm}$ and $700 \mathrm{~nm}$ arrives only at 0.337 and $1.000^{\circ}$, respectively, for incidence angle $70^{\circ}$. This angle change generates a beam spot shift of less than $0.6 \mathrm{~mm}$ on a rhomb face in our set-up. As a result, this can be the reason for the diffracted order superposition on the detector.

To support a more real situation, an ultrathin $4 \mathrm{~nm}$ thick buffer layer of $\mathrm{SiO}_{2}$ is included in the modeling. Let us remember that this buffer layer causes an increase in the computed parameter $\psi$. In the opposite way it influences the values of $\Delta$. In conclusion, this means that for $2 \mathrm{~nm} \mathrm{SiO}$ layer the modeled data will be close to the experimental outputs.

The comparison of spectral ellipsometry angles with those obtained by CWM supports the idea of spreading the mathematical approaches applied to standard optical gratings to the periodic structures typical of microelectronics circuits.

Acknowledgments - This research was partially supported by Grant Agency of Czech Republic - contracts Nos. 202/01/0077 and 105/01/0168 - and by Czech Ministry of Education, Youth and Sports - KONTAKT projects Nos. ME 507 and ME 508.

\section{References}

[1] Shen J., Kirschner J., Surf. Sci. 500 (2002), 300.

[2] Kortright J.B., Awschalom D.D., Stohr J., Bader S.D., Idzerda Y.U., Parkin S.S.P., SChuller I.K., Siegmann H.-C., J. Magn. Magn. Mater. 207 (1999), 7.

[3] Martin J.I., Costa-Kramer J.L., Briones F., Vicent J.L., J. Magn. Magn. Mater. 221 (2000), 215.

[4] Schider G., Krenn J.R., Gotschy W., Lamprecht B., Ditlbacher H., Leitner A., Aussenegg F.R., J. Appl. Phys. 90 (2001), 3825.

[5] Schmitte T., Schwobken O., Goek S., Westerholt K., Zabel H., J. Magn. Magn. Mater. 240 (2002), 24.

[6] Mattheis R., Berkov D., Gorn N., J. Magn. Magn. Mater. 1998-1999 (1999), 216.

[7] Vavassori P., Metlushko V., Grimsditch M., Ilic B., Neuzil P., Kumar R., Phys. Rev. B 61 (2000), 5895. 
[8] Yasumoto K., Yoshitomi K., IEEE Trans. Antennas and Propagation 47 (1999), 1050.

[9] Watanabe K., Petit R., Neviere M., J. Opt. Soc. Am. A 19 (2002), 325.

[10] Popov E., Neviere M., J. Opt. Soc. Am. A 18 (2001), 2886

[11] Li L., J. Opt. Soc. Am. A 13 (1996), 1024.

[12] Ibidem, p. 1870.

[13] Noponen E., Turunen J., J. Opt. Soc. Am. A 11 (1994), 2494.

[14] VišñovskÝ Š., Yasumoto K., Czech. J. Phys. 51 (2001), 229.

[15] Glytsis E.N., J. Opt. Soc. Am. A 19 (2002), 702.

[16] Pagani Y., Van Labeke D., Guizal B., Vial A., Baida F., Opt. Commun. 209 (2002), 237.

[17] Vergohl M., Malkomes N., Staedler T., Matthée T., Richter U., Thin Solid Films 351 (1999), 42.

[18] Bhattacharyya D., Sahoo N.K., Thakur S., Das N.C., Vacuum 60 (2001), 419.

[19] Vergohl M., Malkomes N., Matthée T., Brauer G., Thin Solid Films 377-378 (2000), 43.

[20] Collins R., W., Кон J., Fujiwara H., Rovira P.I., Ferlauto A.S., Zapien J.A., Wronski C.R., Messier R., Apll. Surf. Sci. 154-155 (2000), 217.

[21] Vlček J., Piśtora J., Ciprian D., Yamaguchi T., Postava K., J. Magn. Soc. Jap. 2 (2002) - in print.

[22] Vlček J., Piśtora J., Ciprian D., Yamaguchi T., Vávra I., [In] Proc. XIII Polish-Czech-Slovak Optical Conference Wave and Quantum Aspects of Contemporary Optics, Krzyżowa, Poland, September 9-13, 2002 (to be published).

[23] AzZam R.M.A., Bashara N.M., Ellipsometry and Polarized Light, North Holland, Amsterdam, New York, Oxford, 1977.

[24] Palik E.D., Handbook of Optical Constants of Solids, Academic Press, San Diego, London, Boston 1998. 